\title{
Properties of Exotic Charmonium-like States at CDF
}

\author{
Kai $\mathrm{Yi}^{1}$ for the CDF Collaboration \\ 1- University of Iowa - Dept of Physics and Astronomy \\ Iowa City, IA 52242-1489
}

\begin{abstract}
We report the recent evidence for a new narrow structure, $Y(4140)$, decaying to the $J / \psi \phi$ final state, in exclusive $B^{+} \rightarrow J / \psi \phi K^{+}$decays in a data sample corresponding to an integrated luminosity of $2.7 \mathrm{fb}^{-1}$ collected at the CDF II detector. This narrow structure with its mass well above open charm pairs is unlikely to be a candidate for a conventional charmonium state. From a study of the $X(3872)$ mass and width based on the world's largest sample of $X(3872) \rightarrow J / \psi \pi^{+} \pi^{-}$decays, we find that our $X(3872)$ signal is consistent with a single state, and leads to the most precise measurement of the $X(3872)$ mass.
\end{abstract}

\section{Introduction}

The existence of exotic mesons beyond $q \bar{q}$ has been discussed for 30 years [1, but evidence for such mesons has not been clearly established. The recently discovered states that have charmonium-like decay modes 2 but are difficult to place in the overall charmonium system have introduced challenges to conventional $q \bar{q}$ meson model. The possible interpretations beyond $q \bar{q}$ such as hybrid $(q \bar{q} g)$ and four-quark states $(q \bar{q} q \bar{q})$ have revitalized interest in exotic mesons in the charm sector [3]. So far the observed states involve only $c$ quark and light quark $(u, d)$ decay products, however, the $J / \psi \phi$ final state is a good channel for an exotic meson search which extends to $c$ quark and heavy $s$ quark decay products. An investigation of the $J / \psi \phi$ system produced in exclusive $B^{+} \rightarrow J / \psi \phi K^{+}$decays with $J / \psi \rightarrow \mu^{+} \mu^{-}$and $\phi \rightarrow K^{+} K^{-}$is reported in this note. This analysis is based on a data sample of $\bar{p} p$ collisions at $\sqrt{s}=1.96 \mathrm{TeV}$ with an integrated luminosity of $2.7 \mathrm{fb}^{-1}$ collected by the CDF II detector at the Tevatron. Charge conjugate modes are included implicitly in this note.

It has been six years since the discovery of $X(3872)$, however, the nature of this state has not been clearly understood yet. Due to the closeness of the $X(3872)$ mass with the threshold of $D^{0}$ and $D^{* 0}$, the $X(3872)$ has been proposed as a molecule composed of $D^{0}$ and $D^{* 0}$. The $X(3872)$ has also been speculated to be two states nearby, as in some models like the diquarkantidiqurk model. It is critical to make precise measurements of the mass and width of $X(3872)$ to understand its nature. The large $X(3872) \rightarrow J / \psi \pi^{+} \pi^{-}$sample accumulated at CDF enables us to test the hypothesis of that the $X(3872)$ is composed of two states and to make a precise mass

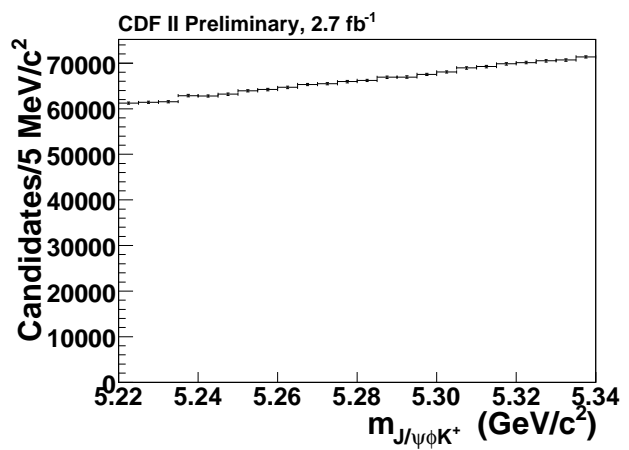

Figure 1: The $J / \psi \phi K^{+}$mass before minimum $L_{x y}\left(B^{+}\right)$and kaon $L L R$ requirements. measurement of $X(3872)$ if it is consistent with one state hypothesis.

DIS 2009 


\section{Evidence of $\mathrm{Y}(4140)$}

We first reconstruct $B^{+} \rightarrow J / \psi \phi K^{+}$signal and then search for structures in the $J / \psi \phi$ mass spectrum [4]. The $J / \psi \rightarrow$ $\mu^{+} \mu^{-}$events are recorded using a dedicated dimuon trigger. The $B^{+} \rightarrow J / \psi \phi K^{+}$candidates are reconstructed by combining a $J / \psi \rightarrow \mu^{+} \mu^{-}$candidate, a $\phi \rightarrow K^{+} K^{-}$ candidate, and an additional charged track. Each track is required to have at least 4 axial silicon hits and have a transverse momentum greater than $400 \mathrm{MeV} / c$. The reconstructed mass of each vector meson candidate must lie within a suitable range from the nominal values $\left( \pm 50 \mathrm{MeV} / c^{2}\right.$ for the $J / \psi$ and $\pm 7 \mathrm{MeV} / c^{2}$ for the $\left.\phi\right)$. In the final $B^{+}$reconstruction the $J / \psi$ is mass constrained, and the $B^{+}$candidates must have $p_{T}>4 \mathrm{GeV} / c$. The $P\left(\chi^{2}\right)$ of the mass- and vertex-constrained fit to the $B^{+} \rightarrow J / \psi \phi K^{+}$candidate is required to be greater than $1 \%$.

To suppress combinatorial background, we use $d E / d x$ and TOF information to identify all three kaons in the final state. The information is summarized in a log-likelihood ratio $(L L R)$, which reflects how well a candidate track can be positively identified as a kaon relative to other hadrons. In addition, we require a minimum $L_{x y}\left(B^{+}\right)$for the $B^{+} \rightarrow J / \psi \phi K^{+}$candidate, where $L_{x y}\left(B^{+}\right)$ is the projection onto $\vec{p}_{T}\left(B^{+}\right)$of the vector connecting the primary vertex to the $B^{+}$decay vertex. The $L_{x y}\left(B^{+}\right)$and $L L R$ requirements for $B^{+} \rightarrow J / \psi \phi K^{+}$are then chosen to maximize $S / \sqrt{S+B}$, where $S$ is the number of $B^{+} \rightarrow J / \psi \phi K^{+}$signal events and $B$ is the number of background events implied from the $B^{+}$sideband. The requirements obtained by maximizing $S / \sqrt{S+B}$ are $L_{x y}\left(B^{+}\right)>500 \mu \mathrm{m}$ and $L L R>0.2$.

The invariant mass of $J / \psi \phi K^{+}$, after $J / \psi$ and $\phi$ mass window requirements, and before and after the minimum $L_{x y}\left(B^{+}\right)$and kaon $L L R$ requirements, are shown in Fig. 11and Fig. 2 , respectively. We do not see $B^{+}$signal at all before the $L_{x y}\left(B^{+}\right)$and kaon $L L R$ requirements, but we see clear $B^{+}$signal after the requirements. A fit with a Gaussian signal function and a flat background function to the mass spectrum of $J / \psi \phi K^{+}$(Fig. 2) returns a $B^{+}$signal of $75 \pm 10$ (stat) events. The $L_{x y}\left(B^{+}\right)$and $L L R$ requirements reduce the background by a factor of approximately 20000 while keeping a signal efficiency of approximately $20 \%$. We

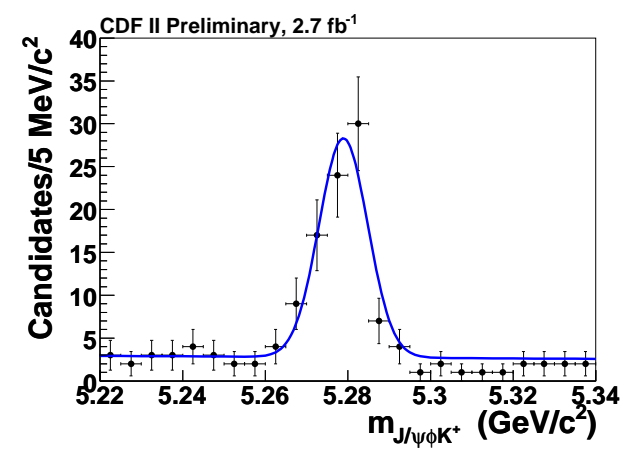

Figure 2: The $J / \psi \phi K^{+}$mass after minimum $L_{x y}\left(B^{+}\right)$and $L L R$ requirements; the solid line is a fit to the data with a Gaussian signal function and flat background function.

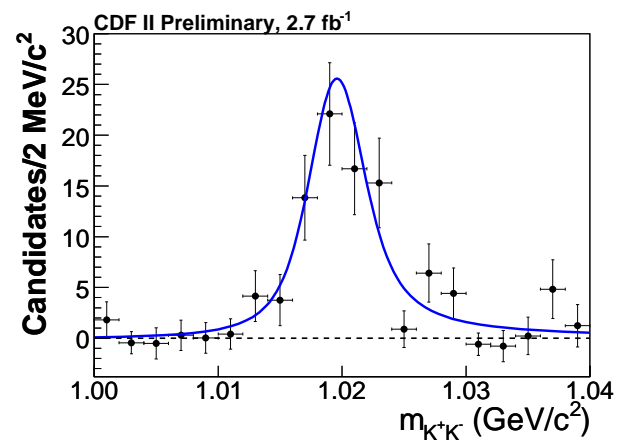

Figure 3: $\quad$ The $B^{+}$sideband-subtracted $K^{+} K^{-}$mass without the $\phi$ mass window requirement. The solid curve is a $P$-wave relativistic Breit-Wigner fit to the data. 
select $B^{+}$signal candidates with a mass within $3 \sigma$ of the nominal $B^{+}$mass; the purity of the $B^{+}$signal in that mass window is about $80 \%$.

The combinatorial background under the $B^{+}$peak includes $B$ hadron decays such as $B_{s}^{0} \rightarrow \psi(2 S) \phi \rightarrow J / \psi \pi^{+} \pi^{-} \phi$, in which the pions are misidentified as kaons. However, background events with misidentified kaons cannot yield a Gaussian peak at the $B^{+}$mass consistent with the 5.9 $\mathrm{MeV} / c^{2}$ mass resolution. Figure 3 shows the $K^{+} K^{-}$mass from $\mu^{+} \mu^{-} K^{+} K^{-} K^{+}$candidates within $\pm 3 \sigma$ of the nominal $B^{+}$mass with $B$ sidebands subtracted and before applying $\phi$ mass window requirement. Using a smeared $P$-wave relativistic Breit-Wigner (BW) [5] line-shape fit to the spectrum returns a $\chi^{2}$ probability of $28 \%$. This shows that the $B^{+} \rightarrow J / \psi K^{+} K^{-} K^{+}$final state is well described by $J / \psi \phi K^{+}$.

We then examine the effects of detector acceptance and selection requirements using $B^{+} \rightarrow J / \psi \phi K^{+} \mathrm{MC}$ events simulated by a phase space distribution. The MC events are smoothly distributed in the Dalitz plot and in the $J / \psi \phi$ mass spectrum. No artifacts were observed from $\mathrm{MC}$ events. Figure 4 shows the Dalitz plot of $m^{2}\left(\phi K^{+}\right)$ versus $m^{2}(J / \psi \phi)$, and Fig. 5 shows the mass difference, $\Delta M=m\left(\mu^{+} \mu^{-} K^{+} K^{-}\right)-$ $m\left(\mu^{+} \mu^{-}\right)$, for events in the $B^{+}$mass window in our data sample. We examine the enhancement in the $\Delta M$ spectrum just above $J / \psi \phi$ threshold. We exclude the highmass part of the spectrum beyond 1.56 $\mathrm{GeV} / c^{2}$ to avoid combinatorial backgrounds that would be expected from misidentified $B_{s}^{0} \rightarrow \psi(2 S) \phi \rightarrow\left(J / \psi \pi^{+} \pi^{-}\right) \phi$ decays. We model the enhancement by an $S$-wave rela-

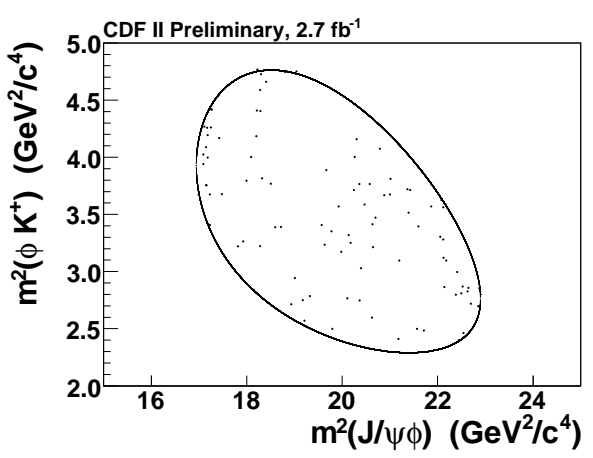

Figure 4: The Dalitz plot of $m^{2}\left(\phi K^{+}\right)$versus $m^{2}(J / \psi \phi)$ in the $B^{+}$mass window. The boundary shows the kinematic allowed region.

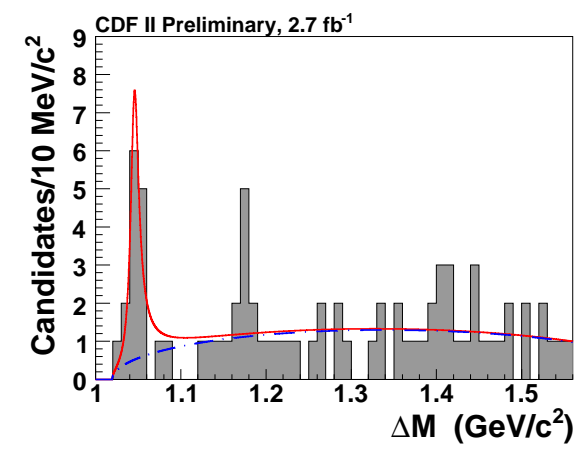

Figure 5: The mass difference, $\Delta M$, between $\mu^{+} \mu^{-} K^{+} K^{-}$and $\mu^{+} \mu^{-}$, in the $B^{+}$mass window. The dash-dotted curve is the background contribution and the red solid curve is the total unbinned fit.

tivistic BW function [6] convoluted with a Gaussian resolution function with the RMS fixed to $1.7 \mathrm{MeV} / c^{2}$ obtained from $\mathrm{MC}$, and use three-body phase space [1] to describe the back-

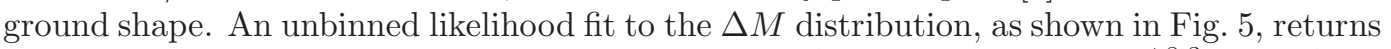
a yield of $14 \pm 5$ events, a $\Delta M$ of $1046.3 \pm 2.9 \mathrm{MeV} / c^{2}$, and a width of $11.7_{-5.0}^{+8.3} \mathrm{MeV} / \mathrm{c}^{2}$.

We use the $\log$-likelihood ratio of $-2 \ln \left(\mathcal{L}_{0} / \mathcal{L}_{\text {max }}\right)$ to determine the significance of the enhancement, where $\mathcal{L}_{0}$ and $\mathcal{L}_{\max }$ are the likelihood values for the null hypothesis fit and signal hypothesis fit. The $\sqrt{-2 \ln \left(\mathcal{L}_{0} / \mathcal{L}_{\max }\right)}$ value is 5.3 for a pure three-body phase space background shape assumption. We generate $\Delta M$ spectra using the background distribution alone, and search for the most significant fluctuation with $\sqrt{-2 \ln \left(\mathcal{L}_{0} / \mathcal{L}_{\max }\right)} \geq 5.3$ in each 
spectrum in the mass range of 1.02 to $1.56 \mathrm{GeV} / c^{2}$, with widths in the range of 1.7 (detector resolution) to $120 \mathrm{MeV} / c^{2}$ (ten times the observed width).

The resulting $p$-value from 3.1 million simulations is $9.3 \times 10^{-6}$, corresponding to a significance of $4.3 \sigma$. We repeat this process with a flat combinatorial non-B background and three-body PS for non-resonance $B$ background and we still get a significance of $3.8 \sigma$.

One's eye tends to draw to a second cluster of events around $1.18 \mathrm{GeV} / c^{2}$ in Fig. 囵, or around $4.28 \mathrm{GeV} / c^{2}$ in $J / \psi \phi$ mass as shown in Fig. 6. It is close to one pion mass above the peak at the $J / \psi \phi$ threshold. However, this cluster is statistically insufficient to infer the presence of a second structure. To investigate possible reflections, we examine

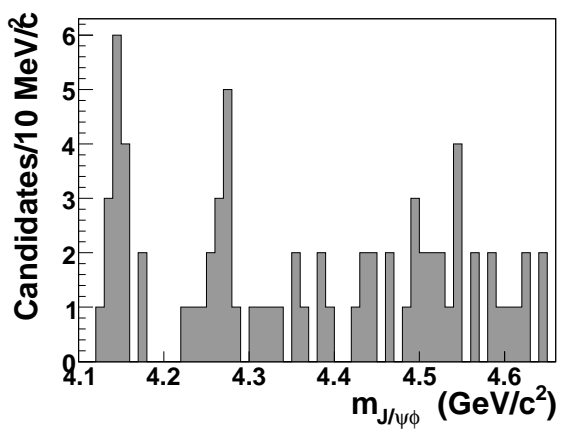
the Dalitz plot and projections into $\phi K^{+}$ and $J / \psi K^{+}$spectrum. We find no evidence for any other structure in the $\phi K^{+}$and $J / \psi K^{+}$ spectrum.

\section{Measurement of the mass of $X(3872)$}

In studying our $X(3872)$ sample we tested the hypothesis of whether the observed signal stems from two different states, as predicted in four-quark models. We fit the $X(3872)$ mass signal with a BW function convoluted with a resolution function [7. Both functions contain a width scale factor that is a free parameter in the fit and therefore sensitive to the shape of the mass signal. The measured width scale factor is compared to the values seen in pseudo experiments which assume two states with given mass difference and ratio of events. The resolution in the simulated events is corrected for the difference between data and simulation measured for the $\psi(2 S)$. The result of this hypotheses test shows that the data is completely consistent with a single state. Under the assumption of two states with equal amount of observed events, we set a limit of $\Delta m<3.2(3.6)$

$\mathrm{MeV} / \mathrm{c}^{2}$ at $90 \%(95 \%)$ C.L. The limit for other ratios of events in the two peaks is shown in Fig. 7 .

Since our signal is consistent with one peak, we proceed and measure the $\mathrm{X}(3872)$ mass

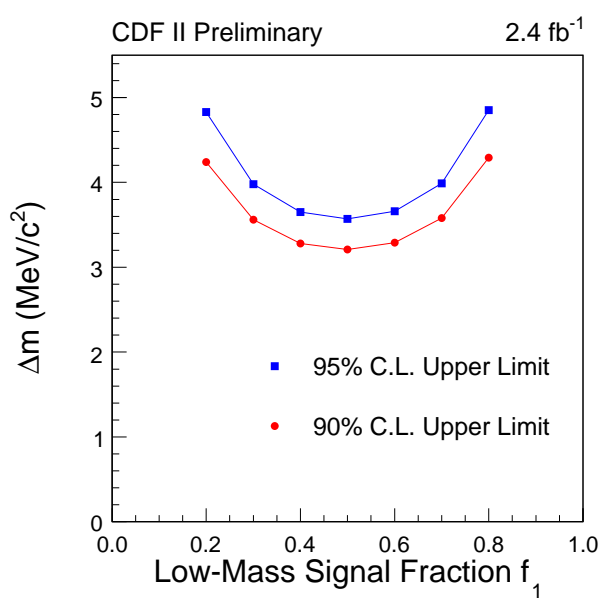

Figure 7: Dependence of the upper limit on the mass difference $\Delta m$ between two states on the low-mass state signal fraction $f_{1}$. 
in an unbinned maximum likelihood fit. The systematic uncertainties are determined from the difference between the measured $\psi(2 S)$ mass and its world average value, the potential variation of the $\psi(2 S)$ mass as a function of kinematic variables, and the difference in $\mathrm{Q}$ value between $X(3872)$ and $\psi(2 S)$. Systematic effects due to the fit model are negligible. The measured $X(3872)$ mass is: $m(X(3872))=3871.61 \pm 0.16($ stat $) \pm 0.19($ syst $) \mathrm{MeV} / c^{2}$, which is the most precise measurement to date as shown in Fig. 8 .

\section{Summary}

The large sample of $B^{+} \rightarrow J / \psi \phi K^{+}$decays enables us to search for structure in the $J / \psi \phi$ mass spectrum, and we find evidence for a narrow structure near the $J / \psi \phi$ threshold with a significance estimated to be at least 3.8 $\sigma$. Assuming an $S$-wave relativistic BW, the mass (adding $J / \psi$ mass) and width of this structure, including systematic uncertainties, are measured to be $4143.0 \pm 2.9$ (stat) \pm 1.2 (syst) $\mathrm{MeV} / c^{2}$ and $11.7_{-5.0}^{+8.3}$ (stat) \pm 3.7 (syst) $\mathrm{MeV} / c^{2}$, respectively. This structure does not fit conventional expectations for a charmonium state because it is expected to have a tiny BR to $J / \psi \phi$ with its mass well beyond open charm pairs. We term it the $Y(4140)$.

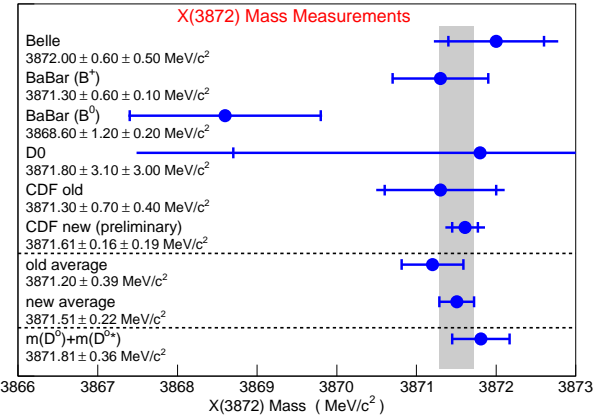

Figure 8: An overview of he measured $X(3872)$ masses from the experiments observing the $X(3872)$.

Studies using our $X(3872)$ sample, the largest in the world, indicate that the $X(3872)$ is consistent with one state hypothesis and this leads to the most precise mass measurement of $(X 3872)$ based on the one state hypothesis. The value is below, but within uncertainties of the $D^{* 0} D^{0}$ threshold. The explanation of the $\mathrm{X}(3872)$ as a bound $\mathrm{D}^{*} \mathrm{D}$ system is therefore still an option.

\section{References}

[1] C. Amsler et al. (Particle Data Group), Phys. Lett. B 667, 1 (2008).

[2] S.-K. Choi et al. (Belle Collaboration), Phys. Rev. Lett. 91, 262001 (2003); D. Acosta et al. (CDF Collaboration), Phys. Rev. Lett. 93, 072001 (2004); S.-K. Choi et al. (Belle Collaboration), Phys. Rev. Lett. 94, 182002 (2005); B. Aubert et al. (BABAR Collaboration), Phys. Rev. Lett. 95, 142001 (2005); S. Godfrey and S. L. Olsen, Ann. Rev. Nucl. Part. Sci 58 (2008) 51.

[3] E. Eichten, S. Godfrey, H. Mahlke, and J. Rosner, Rev. Mod. Phys. 80, 1161 (2008); S. L. Zhu, Phys. Lett. B 625, 212 (2005); F. Close and P. Page, Phys. Lett. B 628, 215 (2005); E. S. Swanson, Phys. Lett. B 588, 189 (2004); L. Maiani, F. Piccinini, A. D. Polosa, and V. Riquer, Phys. Rev. D 72, 031502(R) (2005); N. V. Drenska, R. Faccini, and A. D. Polosa, arXiv:hep-ph/0902.2803v1.

[4] T. Aaltonen et al. CDF Collaboration, Phys. Rev. Lett. 102, 242002 (2009).

[5] B. Aubert et al. (BABAR Collaboration), Phys. Rev. D 78, 071103 (2008).

[6] $\frac{d N}{d m} \propto \frac{m \Gamma(m)}{\left(m^{2}-m_{0}^{2}\right)^{2}+m_{0}^{2} \Gamma^{2}(m)}$, where $\Gamma(m)=\Gamma_{0} \frac{q}{q_{0}} \frac{m_{0}}{m}$, and the 0 subscript indicates the value at the peak mass.

[7] CDF Collaboration, CDF public note 9454; http://www-cdf.fnal.gov/physics/new/bottom/bottom.html 